\title{
Framework for E-Learning Materials Optimization
}

\author{
https://doi.org/10.3991/ijet.v15i11.12721 \\ Dominik Halvoník $\left.{ }^{凶}\right)$, \\ Philosopher University in Nitra, Nitra, Slovakia \\ dominik.halvonik@ukf.sk \\ Jozef Kapusta \\ Philosopher University in Nitra, Nitra, Slovakia \\ Pedagogical University of Cracow, Cracow, Poland
}

\begin{abstract}
Creating educational materials (activities, e-books etc.) in each elearning course can be divided into 2 main parts. The first can be defined as a compilation of ideas and information that we want to pass on to the student. This section of building e-learning materials process is very abstract and correct selection of what we want to teach the students is highly delicate and depends on teacher's skills and didactic principles. The second phase is also important but it can be formalized. Main aim of this paper is to define and confirms a set of formal rules compiled into framework which can be used as a tool for building e-learning materials. We assume that the rules presented in this paper can be used for each e-learning platform. To confirm validity of defined rules we integrated these rules into module on LMS Moodle and part of this paper is proposed experiment carried out on the same platform.
\end{abstract}

Keywords - E-learning, formal rules, structural evaluation, framework, Moodle

\section{$1 \quad$ Introduction}

E-learning usage in today's learning is essential part on most of the organizations such as universities, other state funded institutes intended for learning and companies that can be defined as 'learning organizations' [1]. Each e-learning platform is useless without content, which can be presented to students. This content is basically the most important part of the entire e-learning process because these materials are the direct source of information which is used for learning. One of the problems that e-learning is facing at the moment is the creation of quality educational material. We have many tools and LMS (Learning Management System) or VLE (Virtual Learning Environment) that helps us manage and control the process of learning via e-learning. These platforms and tools can be considered as very powerful and in terms of learning process management [2]. The main problem is the content itself. In most cases the learning materials such as e-books or interactive activities are mostly created by teachers them self [3]. On the other hand there are cases when teachers can reach institutions responsible for emitting these materials. For example, in Slovakia the institution responsible for solving these requests is State Educational Institute. 
However, obtaining learning materials this way is time consuming and not really effective, especially in fields like IT where teacher have to keep up with new technologies emerging almost every day. So waiting for separate institution to adapt the new technology, create and distributes learning materials for diverse e-learning platforms is not really an option [4]. That brings us back to the first way of obtaining the learning materials, teachers have to create the materials by them self. The main emphasis in this paper is to define formalized set of rules improves the structural nature of educational materials. In our article we will focus on two main parts. First one is the rule definition based on previous research. Second one is the design of experiment which should confirm the validity of proposed rules. To achieve relevant results, it's crucial to follow all standardized steps defined in selected methodology.

The experimental method (or briefly the experiment) has a key position among the methods of quantitative research. This is because, as the only research method, it can prove the causal consequences of pedagogical activity. It is able to demonstrate how one educational phenomenon affects another. Using other research methods, we can only identify the interrelationships of phenomena in pedagogy, but we cannot confirm with certainty that these relationships are causal [5].

Our paper aims to define set of rules and compile them into one framework which can be used for building any learning material which will be used via e-learning. The framework will be then implemented into software solution which will be used as tool in experimental phase. Also, in this paper we will focus on empirically prove that the defined formal rules for learning materials creation improves the students learning experience.

\section{$2 \quad$ Literature Review}

Studies clearly demonstrate that the integration of e-learning into the education process has a clear positive impact and increases the effectiveness of education [6]. It is also true, that e-learning as a whole is a demanding process and the structural form of the course itself is many times not the key factor for course success [7]. That means that even courses designed in a very simple way, for example if course is composed only from links to external resources, can be considered very successful by students if there is a strong focus on discussion and activity. This is confirmed by the increasing trend of interest in microlearning courses, gamming, etc., i.e. content structures that are different from the "ordinary" concept of e-learning courses [8].

On the other hand student's attention is very influenced by the graphic and content processing of the educational material, with the help of which it acquires the required knowledge [9]. This fact can be also observer, even indirectly, on many studies focused on conducted student behavior in VLE [10]. Based on these findings we assume, that even when the formal representation of the content is not the key factor which defines if the course will or will not be successful from the student's level of understanding point of view, it is still a vital part which cannot be underestimated when the course is designed. 
Nowadays, we can find several methodologies which are focused on this problematic. There are frameworks where main criterion is the students result in final exam after finishing the course [11]. We can also use methodical guidelines like "Methodological Issues of Textbook Assessment" from Hrehovcik [12], that are focused strictly on the subject of the content and based on that modifies the rules. One of the most detailed methodologies has been introduced by Klement in 2011. In this methodology the author is focusing strictly on form, layout and content structure of e-learning course. Teacher (author) has to create a learning material rich in a range of formal and content elements that will fully replace present interpretation and allow understanding and memorization of study content. The basic requirements for the study text for distance learning in the form of e-learning are [13]:

- Text clarity

- Dividing the text into shorter study units

- A large number of activating and motivating elements (multimedia elements in static or dynamic form)

- Provide a large number of specific examples and practical examples of content application

- Applying a large number of feedback elements

- Study guides

- Orientation and explanation apparatus (navigation icons, margins, color classification, font type, etc.)

As you can see all of the presented rules are quite general. Most of the methodologies and frameworks that we encountered have the same problem. It is very hard to unify them into machine applicable algorithm which can be used for any electronically input. Partial success in terms of methodology algorithmization focusing on formal criteria has been achieved by Rohlikova, who described the graphic processing of the educational content as follows:

- Each page has to have the same text layout

- Throughout the document, there have to be used similar font (size, style etc.)

- Each page has to contain some text supplements (illustrations, photographs, tables, charts, diagrams, diagrams, maps, etc.).

\section{$3 \quad$ Methodology}

Based on the previously done research we extract and formulate set of formal rules (Table 1).

The rules are divided into two main groups:

1. Repeating rules

2. One-time applicable rules.

The main logic of usage for these rules is that each learning material has to be divided into pages. Even when it is a 1-page document the algorithm will be applied the same. 
Table 1. List of rules

\begin{tabular}{|c|l|c|l|}
\hline No. & \multicolumn{1}{|c|}{ Rule name } & $\begin{array}{c}\text { Number of } \\
\text { points }\end{array}$ & \multicolumn{1}{|c|}{ Type } \\
\hline 1 & $\begin{array}{l}\text { The maximum number of words without multimedia content } \\
\text { per page is } 150 \text { - 250 }\end{array}$ & 4 & Repeating rule \\
\hline 2 & $\begin{array}{l}\text { The minimum number of words without multimedia content } \\
\text { per page is 20-30 }\end{array}$ & 2 & One-time applicable rule \\
\hline 3 & One multimedia unit for 150 - 250 words per page & 3 & Repeating rule \\
\hline 4 & The maximum number of words per page is 1000 & 3 & One-time applicable rule \\
\hline 5 & Multimedia content type image & 1 & One-time applicable rule \\
\hline 6 & Multimedia content type video & 2 & One-time applicable rule \\
\hline 7 & Interactive multimedia content (flash etc.) & 3 & One-time applicable rule \\
\hline 8 & Using text highlighting: bold & 1 & One-time applicable rule \\
\hline 9 & Do not use underline for highlighting & 1 & One-time applicable rule \\
\hline 10 & $\begin{array}{l}\text { The maximum number of consecutive words highlighted in } \\
\text { italics is30 }\end{array}$ & 2 & Repeating rule \\
\hline 11 & The maximum number of different font types on the page is 2 & 3 & One-time applicable rule \\
\hline 12 & The maximum number of different font sizes on the page is 3 & 3 & One-time applicable rule \\
\hline 13 & Are paragraphs on the page? (if yes) & 2 & One-time applicable rule \\
\hline
\end{tabular}

Each rule has its own logic and it is mostly independent from other rules. For clarity we would like to provide explanation how to implement the rule for each one of presented rules.

Rule no. 1 has to be applied on each page within the e-learning content which contains text. However, it may not exceed a certain maximum limit. This boundary ensures the text clarity and clarity of the learned topic, as well as keeping the student's attention by requiring his / her interaction when going to the next paragraph of educational content after studying the current one. If the page contains a number of words in the range of 150 to 250 words, it is rated 4 points. When you exceed 250 words, 1 point for every 20 words on the page is decreased. On the other hand, if the lower limit of the interval is not reached, a 1-point decrease occurs for every 30 words that are missing on the page. The reason for this rule is to try to achieve the optimal number of words on the page so that the student is not exposed to an absurdly long text without the support of multimedia content, and on the other hand that the educational material is rich enough on the content page to allow the student to focus and concentrate to the presented content.

Rule no. 2 as well as the previous rule, this is also intended to achieve the optimal number of words on the educational content page. If a page has a number of words in a given interval, the page is rated by 2 points. If the page does not contain a minimum number of words, for example 20 , we will give 0 points. If the page exceeds the specified interval, the number of points decreases by every 20 words on the page. The reason why the content will be received 0 points as a result of this rule evaluation after reaching 70 words is that based on our assumption minimal number of words describing specific topic is 150. This rule should eliminate pages of educational content that are 
navigational rather than content in nature. This kind of pages should be minimal within the educational content.

Rule no. 3 aims to guide the creators of individual e-learning materials to make greater use of multimedia elements in the creation of educational materials. There are number of words on the page, which should be supported by multimedia content. If there is 1 multimedia element on the page and the number of words on the page is within the specified interval, the page gets 3 points. If the page contains more words than the upper bound of the interval, it starts as if it were from the beginning. This rule would be exempt from the context of other rules and could produce an unlimited number of points. Therefore, it is important to use the rules as a whole, not separately. Once there is at least 1 multimedia element on the page, but the number of words did not reach the bottom of the interval, the score is reduced by 1 point for every 50 words. We would like to prevent the creation of educational content in such a way that the authors would only put videos or other multimedia elements on individual pages without additional written explanation.

Rule no. 4 is only applicable when combining rules, no. 1 and no. 3. Also, it is inappropriate to include too much written content on the one hand when supporting multimedia content. Therefore, a certain limitation must be given in terms of content. This rule has more Boolean character than the previous rules. Once the above rules are met and the page exceeds the number of words 1000 , this rule assigns 0 points, if the maximum number is adhered to 3 points.

Rules no. 5, 6 and 7 possess the same logic for different type of multimedia content. If the multimedia content is presented on the page, add specific number of points, else do not.

Rule no. 8 is applied when the page includes at least one highlighted text with "bold" style. Aim of this rule is to highlight the key information from the content of the page.

Rule no. 9 this rule has been defined for the sake of readability of text. Using underline as way of highlighting text is a bad practice decreasing readability of written text [14].

Rule no. 10 has similar logic as no. 9. The difference is that this rule does not restricts usage of italic highlighting style, but only reduce it to 30 words. Based on our assumption more words highlighted will cause problems for students in terms of keeping focus on the highlighted text.

Rules no. 11 and 12 are directly extracted from recommendations provided by Rohlikova. The aim of these rules is to make sure that the presented content will have consisted styling and will not provide unnecessary load to student's attention.

Rule no. 13 defines if provided text has been structured into paragraphs. This rule will provide points if there is at least 1 paragraph on the page. 


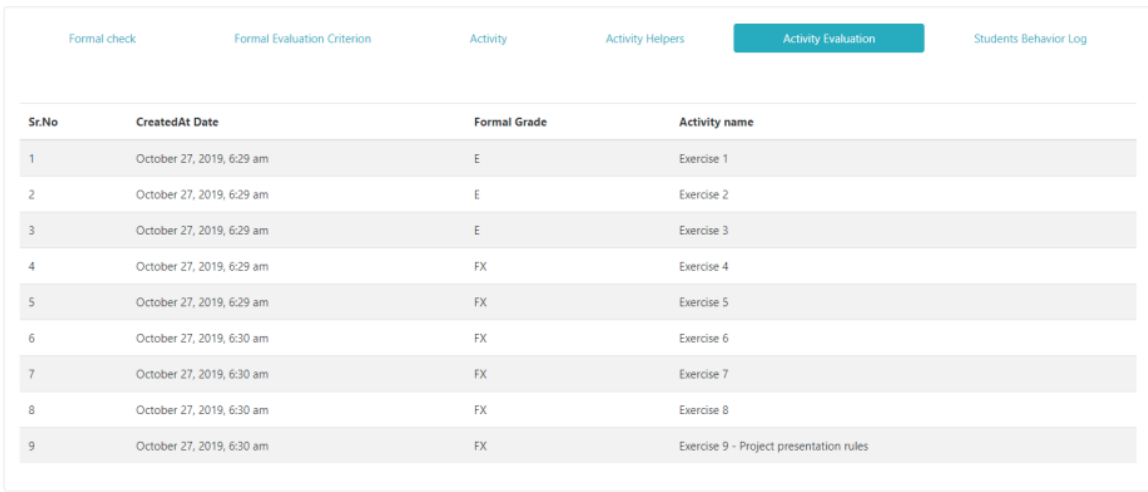

Fig. 1. List of evaluated activities in administration panel in Moodle

As we can see all of the rules can be easily implemented into any programming language as a software tool automatically providing results. For the sake of the experiment that we planned to perform we decided that the platform on which the experiment will take place will be LMS Moodle. So we implemented the defined framework into Moodle module, which has been responsible for evaluating each supported activity of any selected course. By supported activity we mean any activity whose content is stored in the Moodle database (book, page, lesson etc.). So external links, or uploaded PDF files were excluded from the experiment.

Because Moodle is the central platform for teachers to organize their learning resources, the module application should be started from there. In Moodle it is possible to create external tool activities, which start external learning resources that can be anything from a homepage with information, to a comprehensive online course system. To achieve this, Moodle uses the Learning Tools Interoperability Protocol (LTI) which was developed by IMS Global. The LTI protocol standardizes the way of how different e-Learning systems communicate. The Moodle instance that launches the external tool is called "LTI tool consumer" in this context and the external resource that is launched, is called the "LTI tool provider". The module application which is described in more detail in this and in the next section also should implement the LTI protocol and can be started as external tool activity from Moodle (or other LTI consumers). Therefore, the application can be used by any LTI consumer if it was set up for that consumer previously [15]. 


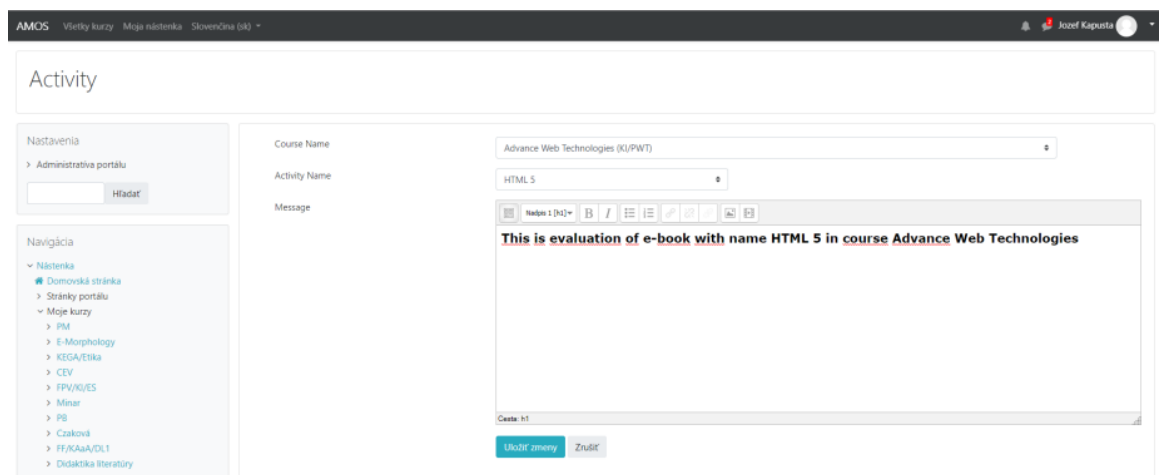

Fig. 2. Form for activity evaluation based on course

When the framework is in use (Fig.3) we are dividing each educational object into pages. Each page is evaluated individually and acts like an independent entity. After each page has been evaluated, we calculate final score based on number of pages and score sum of all pages.

$$
\text { finalScore }=\operatorname{round}\left(\frac{\text { sumOfScoreForEachPage }}{\text { numberOfPages }}\right)
$$

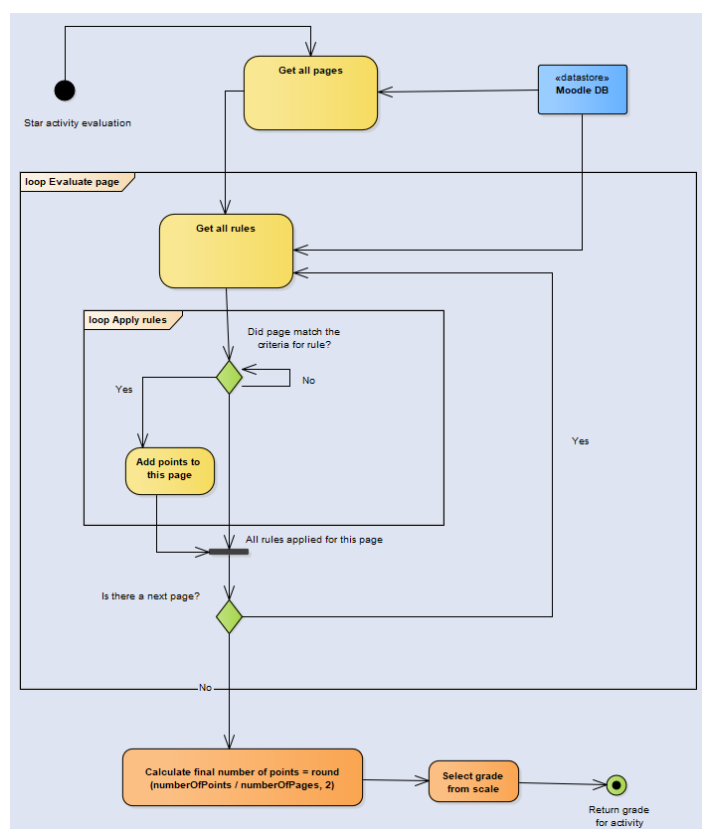

Fig. 3. Diagram representing framework application 
After we obtain the final score for activity, we need to select grade. Based on the description we can tell the teacher if the activity is formatted well or if it needs improvement. We have drawn up the evaluation scale on which we can present the structural correctness or the inadequacy of the given educational material, taking into account aspects of pedagogical research dealing with the creation and evaluation of evaluation scales. For our needs, we choose the interval scale [16], using the overall rating. The scale listed above has the following structure:

Table 2. Scale structure

\begin{tabular}{|c|c|l|}
\hline Points & Rating & \multicolumn{1}{|c|}{ Description } \\
\hline $\begin{array}{c}40 \text { and } \\
\text { more }\end{array}$ & A & Educational material reaches the highest possible level \\
\hline $32-39$ & B & There are minor shortcomings in the educational material \\
\hline $25-31$ & C & Educational material contains certain shortcomings requiring the author's attention \\
\hline $19-24$ & D & $\begin{array}{l}\text { Educational material has several drawbacks. The author is recommended to modify the } \\
\text { content }\end{array}$ \\
\hline $11-18$ & E & $\begin{array}{l}\text { Educational content contains major shortcomings and is recommended complex re- } \\
\text { building }\end{array}$ \\
\hline $0-10$ & FX & $\begin{array}{l}\text { Educational material is structurally deficient in design, which may lead to a decrease its } \\
\text { educational value }\end{array}$ \\
\hline
\end{tabular}

\section{Experiment Preparation}

The experiment as a common tool is significant because it is used to determine the effectiveness of educational activities (educational program, intervention, training, textbooks, etc.). Other research methods cannot directly do this. For example, observation only shows how the educational effect is realized, but not whether it is effective. We can only be convinced of its effectiveness by comparing it with another educational effect - and this can only be done in the form of an experiment. Neither observation, questionnaire, interview, scaling, sociometry, or test have the potential of an experiment - these research methods are used to describe educational reality, or to describe its change, but not to determine its effectiveness. Experiment is therefore an indispensable research method in introducing educational changes and innovations and in carrying out transformations or reforms.

An experiment is a comprehensive research method, since other research methods must inevitably be used to obtain data on subjects of the experiment - test, questionnaire, observation, scaling, sociometry, etc. We measure the characteristics of subjects - both before and after the experiment. Such research uses a group of research methods that are framed by experiment.

There is a wide variety of educational processes using experiments as a tool in teaching-indeed these are as many as the detailed science and engineering objectives to be taught. Practically, however, all these fall into two general approaches: virtual experiments and real ones, both being in the centre of interest, didactically [17]. In our case we decided to choose the real one. Our experiment will take place on Faculty of Natural Science, University of Constantine the Philosopher. Specifically, we will lead this 
experiment on course with name Advance Web Technologies. Students on this course will use LMS Moodle as a source of all information. We will dive our students into 2 separate groups randomly. These groups will be names group A and group B. Group A will have only access to course activities developed without usage of our framework. Group B on the other hand will have access only to activities which have been modified based on the results obtained after applying the results of structural framework. The content in both versions is the same.

The experiment will start at the beginning of semester and it will last until the end of semester. Both groups will not be informed that there are structural differences in their activities. Based on previous years we assume that number of students involved into this experiment will be around 40. This assumption is made based on number of students assigned to this course in last 4 years. The developed module activated on LMS Moodle will also monitor behavior of each student on each activity. Based on the obtained data and students results from the final exam from this course we will confirm if the application of proposed framework provides benefits from the students learning experience point of view.

\section{$5 \quad$ Discussion and Conclusion}

According to the results of one of the studies [18], 96.50\% (110 people) teaches think that the new web technology tools are useful in education. Only $3.5 \%$ (10 people) answered no. Studies of this type provides strong prediction that e-learning will not be only part of normal learning process but in near feature it will fully replace the current way of teaching / learning. That brings us back to the way how we are creating and designing our educational content.

Based on previous research is clear that at the moment we are missing one unified framework on any unified set of rules which will help teachers and authors of e-learning educational materials translate they required content into correct formal form. We assume that by using suggested framework we are able to clearly and comprehensively evaluate individual e-learning educational materials, such as the electronic book in LMS Moodle or static textbooks in PDF format. The biggest benefit of this framework is its simplicity which helps when the framework is implemented into any type of software equipment. The relevant criteria can be easily implemented in the form of a program that will evaluate the relevant educational materials in electronic form.

The interpretation of the evaluation obtained on the basis of these criteria can also be positively evaluated, given its clarity as well as the combination of the individual criteria. This part of the evaluation is quite important given that there is only a very small number of methodologies which follow the individual criteria. As we have seen in the evaluation criteria used by presented methodologies, the individual criteria are not interrelated, which may lead to the situation that some educational material will appear correct in order of structure, but a certain structural part will be absolutely inadequate. The rules proposed by us will detract from this deficiency.

In the following research we would like to focus on executing the designed experiment and verify the suggested structural framework. The main aim of the experiment is 
to confirm whether students who attended a course that met the criteria proposed by us have achieved better results than students who have used the unadjusted course. We are aware of many data analysis approaches on this occasion, we will use the classic analytical tools of Educational Data Mining [19]. This ensures that we obtain and formulate relevant results with a high degree of accuracy.

\section{$6 \quad$ References}

[1] A.W. Bates, Technology, e-learning and Distance Education, ISBN 9780415284370, Routledge, 2005.

[2] P.A.J. Hsieh, V. Cho, "Comparing e-Learning tools' success: The case of instructor-student interactive vs. self-paced tools," Computers \& Education, vol. 57, issue 3, 2011. https://doi.org/10.1016/j.compedu.2011.05.002

[3] M. Landoni, P. Diaz "Design and Evaluation for Teaching and Learning," E-education: Design and Evaluation, vol 3, 2003.

[4] M. Pomffyova, "E-learning the current state and prospects for the use of technology.," Efocus, vol. 2, 2009.

[5] L.B. Christensen, "Experimental methodology 3rd ed.", ISBN 0205082440, 1985.

[6] P. Mihalov, "E-learning effectiveness.", In ICL, Villach, Austria, 2009, ISBN 978-3-89958481-3, p. 551-557.

[7] M. Munk, D. Munkova, L. Benko, "Identification of Relevant and Redundant Automatic Metrics for MT Evaluation," Multi-disciplinary trends in artificial intelligence., vol. 10053, pp. 141-152, 2016. https://doi.org/10.1007/978-3-319-49397-8_12

[8] C. Dichev, D. Dicheva., "Gamifying education: what is known, what is believed and what remains uncertain: acritical review," International Journal of Edu-cational Technology in Higher Education, vol. 14, 2017. https://doi.org/10.1186/s41239-017-0042-5

[9] L. Rohlikova, "E-learning methodics," In Complite teacher, Plzeň, Czech Republic, 2012.

[10] M. Munk, M. Drlik, "Analysis of stakeholders' behaviour depending on time in virtual learning environment," In Applied Mathematics and Information Sci-ences, Vol. 8, Issue 2, 2014 , ISSN 19350090, p. 773 - 785. https://doi.org/10.12785/amis/080238

[11] M. Daniels, E. Sarte, J. D. Cruz, "Students' perception on e-learning: a basis for the development of e-learning framework in higher education institutions", in The International Conference on Information Technology and Digital Applications, vol. 4822019.

[12] T. Hrehovcik, "Criteria for assessing the quality of textbooks for general education subjects", 2015.

[13] M. Klement, "Approaches to the evaluation of electronic study supports de-signed for the realization of teaching in the form of elearning,", ISBN 978-80-87557-13-6, Velfel Ladislav, 2011.

[14] UNSS, “Do you know what is clear printing?”, Bridges of inclusion vol. 2, 2011.

[15] J. Schweighofer, B. Taraghi, M. Ebner, "Development of a Quiz", International Journal of Emerging Technologies in Learning, vol. 14, no. 23, ISSN: 1863-0383, 2019. https://doi. org/10.3991/ijet.v14i23.11484

[16] P. Gavora, "Electronic textbook of educational research," ISBN 978-80-223-2951-4, Bratislava, 2010.

[17] C. Tsihouridis, D. Vavougios, M. Batsila, G. Ioannidis, "The Optimum Equilibrium when Using Experiments in Teaching - Where Virtual and Real Labs Stand in Science and Engineering Teaching Practice.", International Journal of Emerging Technologies in Learning, vol. 14, no. 23, ISSN: 1863-0383, 2019. https://doi.org/10.3991/ijet.v14i23.10890 
[18] S. Caliskan, Z. Guney, R. G. Sakhieva, D. G. Vasbieva, N. A. Zaitseva, "Teachers' Views on the Availability of Web 2.0 Tools in Education", International Journal of Emerging Technologies in Learning, vol. 14, no. 22, ISSN: 1863-0383, 2019. https://doi.org/10.3991/ ijet.v14i22.11752

[19] M. Drlik, P. Svec, J. Skalka, "Comparison of Approaches to the Data Analysis in the Virtual Learning Environments.", In DIVAI, Štúrovo, Slovakia, 2014, ISBN 978-80-7478-497-2, p. $561-572$.

\section{$7 \quad$ Authors}

Dominik Halvoník is with Department of Informatics Constantine the Philosopher University in Nitra, Nitra, Slovakia.

Jozef Kapusta is with Department of Informatics Constantine the Philosopher University in Nitra, Nitra, Slovakia. He also works at Institute of Computer Science, Pedagogical University of Cracow, Cracow, Poland. jkapusta@ukf.sk, jkapusta@up.krakow.pl

Article submitted 2019-12-14. Resubmitted 2020-02-10. Final acceptance 2020-02-12. Final version published as submitted by the authors. 